\title{
A GAUSS-KUZMIN-LÉVY THEOREM FOR A CERTAIN CONTINUED FRACTION
}

\author{
HEI-CHI CHAN
}

Received 2 April 2003

\begin{abstract}
We consider an interval map which is a generalization of the well-known Gauss transformation. In particular, we prove a result concerning the asymptotic behavior of the distribution functions of this map.
\end{abstract}

2000 Mathematics Subject Classification: 11J70, 37L40, 60A10.

1. Introduction. In 1800, Gauss studied the following problem. In modern notation, it reads as follows. Write $x \in[0,1)$ as a regular continued fraction

$$
\frac{1}{a_{1}+\frac{1}{a_{2}+\cdots}}:=\left[a_{1}, a_{2}, \ldots\right]_{G} .
$$

Here, $a_{k} \in\{1,2, \ldots\}$. Define the map (now known as the Gauss transformation or the continued fraction map) $T_{G}:[0,1) \rightarrow[0,1)$ as follows: for $x=0, T_{G} 0:=0$; for $x \neq 0$, we have

$$
T_{G} x=T_{G}\left[a_{1}, a_{2}, \ldots\right]_{G}:=\left[a_{2}, a_{3}, \ldots\right]_{G} .
$$

Note that $T_{G}$ removes the first digit and the first level of $x=\left[a_{1}, a_{2}, \ldots\right]_{G}$. By $T_{G}$, we generate a sequence of $x_{k}$ in the unit interval with

$$
x_{k}=T_{G}^{k} x_{0}
$$

Assuming that the "seed," $x_{0}$, is a random real number uniformly distributed in the unit interval, define the distribution function

$$
F_{k}(t)=\text { probability that } x_{k} \leq t, \text { for } 0 \leq t \leq 1 \text {. }
$$

In his notebook, Gauss remarked, after some numerical computations, that "they $\left[F_{k}\right]$ come out so complicated that no hope appears to be left." (See Knuth [17, page 346]).

Twelve years later, in a letter he wrote to Laplace, Gauss stated, without proof, that

$$
\lim _{k \rightarrow \infty} F_{k}(x)=\frac{\log (1+x)}{\log 2} .
$$

However, he was unable to describe the behavior of $F_{k}$ for a large but finite $k$. At the time, Gauss considered the study of $F_{k}$ a problem he could not resolve to his satisfaction. 
A century later, a proof was finally provided by Kuzmin [18]. In the same paper, he actually proved that, for large $k$, one has

$$
F_{k}(x)=\frac{\log (1+x)}{\log 2}+r_{n}(x),
$$

where $r_{n}(x)=O\left(q^{\sqrt{n}}\right)$, and $0<q<1$. Around the same time, Lévy [19], by using a different method that employs probabilistic notions, proved that

$$
r_{n}(x)=O\left(q^{n}\right)
$$

Note that in the 60's, Szüsz [27] was able to prove the same result by using Kuzmin's approach. The asymptotic behavior of $F_{k}(x)$ was finally resolved in 1974 by Wirsing [30] and a complete solution to Gauss's problem was found a few years later by Babenko [1]. For detailed introductions, see [6, 8, 10, 11, 12, 13, 14, 15, 16, 17, 23, 25]. For various extensions and generalizations, see $[4,5,9,20,21,22,24,26,28,29]$. See also the monographs [15, 25]. Just as in the original Gauss-Kuzmin-Lévy Problem, the hard part of these generalizations often involves finding the explicit expressions of the distribution functions. Finally, we remark that the Gauss transformation has strong ties with chaos theory $[2,3]$.

In this note, we consider generalization of the Gauss transformation and prove an analogous result. Write $x \in[0,1)$ as

$$
\frac{2^{-a_{1}}}{1+\frac{2^{-a_{2}}}{1+\cdots}}:=\left[a_{1}, a_{2}, \ldots\right] .
$$

Here, $a_{k}$ are natural numbers (see the next section for details) and one should think of $a_{k}$ as the digits of $x$. Define the generalized Gauss transformation $T:[0,1) \rightarrow[0,1)$ as follows: for $x=0, T 0:=0$; for $x \neq 0$, we have

$$
T x=T\left[a_{1}, a_{2}, \ldots\right]:=\left[a_{2}, a_{3}, \ldots\right] .
$$

Here, $T$ removes the first digit and the first level of $x=\left[a_{1}, a_{2}, \ldots\right]$. Note that this is the same as, for $x=\left[a_{1}, a_{2}, \ldots\right] \neq 0$,

$$
T x=\frac{2^{-a_{1}}}{x}-1
$$

Likewise, we can generate a sequence of $x_{k}$ in the unit interval with

$$
x_{k}=T^{k} x_{0}
$$

Assuming that the "seed," $x_{0}$, is a random real number uniformly distributed in the unit interval, define the distribution function

$$
G_{k}(t)=\text { probability that } x_{k} \leq t, \text { for } 0 \leq t \leq 1 \text {. }
$$

Note that $G_{0}(t)=t$. Our main result is the following theorem. 
THEOREM 1.1. Let $c^{-1}=\log (4 / 3)$. Then, there exist

$$
G_{k}(t)=c \log \left(1+\frac{t}{2+t}\right)+O\left(q^{k}\right),
$$

where $0<q<1$.

In Section 2, we set up the necessary machinery, and in Section 3, we prove Theorem 1.1 .

2. Preliminaries. In this section, first, we show in Lemma 2.1 that $x \in[0,1)$ can be written in the form of (1.8).

LEMMA 2.1. For all $x \in[0,1)$, there exist integers $a_{k} \in\{0,1,2, \ldots\}$ such that

$$
x=\frac{2^{-a_{1}}}{1+\frac{2^{-a_{2}}}{1+\cdots}} .
$$

Proof. For any $x \in[0,1)$, we can find a natural number $a_{1}$ such that

$$
\frac{1}{2^{a_{1}+1}}<x \leq \frac{1}{2^{a_{1}}} .
$$

This implies, for some $p \in[0,1)$,

$$
x=(1-p) 2^{-a_{1}}+\frac{p}{2} 2^{-a_{1}}=\left(1-\frac{p}{2}\right) 2^{-a_{1}} .
$$

Defining $x_{1} \in[0,1)$ by $x_{1}=p /(2-p)$, we can write $x$ as

$$
x=\frac{2^{-a_{1}}}{1+x_{1}} .
$$

Since $x_{1} \in[0,1)$, we can repeat the same iteration and obtain

$$
x=\frac{2^{-a_{1}}}{1+\frac{2^{-a_{2}}}{1+\cdots}} .
$$

Thus, the lemma is proven.

We remark that, from the above proof, it follows that the digits $\left\{a_{1}(x), a_{2}(x), \ldots\right\}$ are related by

$$
a_{k}(x)=a_{1}\left(T^{k-1} x\right),
$$

where $a_{1}(x)=m$ if $x \in\left(2^{-m-1}, 2^{-m}\right]$.

Next, we want to prove the convergence of expansion of the type of (2.1). Define $\left[a_{1}, a_{2}, \ldots, a_{n}\right]$, the convergent of $x$, by truncating the expansion on the right-hand side of (2.1). We want to show

$$
x=\lim _{n \rightarrow \infty}\left[a_{1}, a_{2}, \ldots, a_{n}\right]
$$


To this end, define integer-valued functions $P_{n}(x), Q_{n}(x)$ by the following:

$$
\begin{gathered}
P_{k}(x)=2^{a_{k}(x)} P_{k-1}(x)+2^{a_{k-1}(x)} P_{k-2}(x), \quad k \geq 2, \\
Q_{k}(x)=2^{a_{k}(x)} Q_{k-1}(x)+2^{a_{k-1}(x)} Q_{k-2}(x), \quad k \geq 1,
\end{gathered}
$$

with $a_{0}(x)=0, P_{0}(x)=0, P_{1}(x)=1, Q_{-1}(x)=0$, and $Q_{0}(x)=1$.

Standard induction arguments show that

$$
\begin{gathered}
\frac{2^{-a_{1}} \mid}{\mid 1}+\frac{2^{-a_{2}} \mid}{\mid 1}+\cdots+\frac{2^{-a_{k}} \mid}{\mid 1+t}=\frac{P_{k}+t 2^{a_{k}} P_{k-1}}{Q_{k}+t 2^{a_{k}} Q_{k-1}}, \quad 0 \leq t \leq 1, \\
P_{n-1}(x) Q_{n}(x)-P_{n}(x) Q_{n-1}(x)=(-1)^{n} 2^{a_{1}} \cdots 2^{a_{n-1}} .
\end{gathered}
$$

Note that the left-hand side of (2.9) is a compact notation of continued fractions of the type of (1.8) with $k$ levels.

By combining Lemma 2.1 and (2.9), we have, for $x \in[0,1)$,

$$
x=\frac{P_{n}(x)+t 2^{a_{n}(x)} P_{n-1}(x)}{Q_{n}(x)+t 2^{a_{n}(x)} Q_{n-1}(x)},
$$

where $t=T^{n} x$. Taking $t=0$ in (2.11) gives

$$
\left[a_{1}, \ldots, a_{n}\right]=\frac{P_{n}(x)}{Q_{n}(x)} .
$$

By combining (2.10) and (2.12), we have

$$
\left|x-\left[a_{1}, \ldots, a_{n}\right]\right|=\frac{2^{a_{1}} \cdots 2^{a_{n}}}{Q_{n}\left(t^{-1} Q_{n}+2^{a_{n}} Q_{n-1}\right)},
$$

where $t=T^{n} x$. Note that this equation, which measures the difference between $x$ and its convergent, is the key ingredient of the following estimate.

LEMMA 2.2. For all $x \in[0,1)$, there exists $\left|x-\left[a_{1}, \ldots, a_{n}\right]\right| \leq(1 / 2)^{n}$.

REMARKS 2.3. This lemma implies (2.7).

Proof. By using (2.13) and the fact that $t^{-1} \geq 1$, we have

$$
\left|x-\left[a_{1}, \ldots, a_{n}\right]\right| \leq \frac{2^{a_{1}} \cdots 2^{a_{n}}}{Q_{n}\left(Q_{n}+2^{a_{n}} Q_{n-1}\right)}:=s_{n} .
$$

We claim that

$$
s_{n} \leq \frac{1}{2} s_{n-1}
$$

Indeed,

$$
s_{n} \leq \frac{1}{2}\left(\frac{2^{a_{1}} \cdots 2^{a_{n-1}}}{Q_{n} Q_{n-1}}\right) \leq \frac{1}{2}\left(\frac{2^{a_{1}} \cdots 2^{a_{n-1}}}{Q_{n-1}\left(Q_{n-1}+2^{a_{n-1}} Q_{n-2}\right)}\right)=\frac{1}{2} s_{n-1} .
$$

Note that, in obtaining the first inequality, we have used the fact that $Q_{n} \geq 2^{a_{n}} Q_{n-1}$. In obtaining the second inequality, we have used the fact that $Q_{n} \geq Q_{n-1}+2^{a_{n-1}} Q_{n-2}$. 
This proves the claim. By direct computation, we have $s_{1}=2^{-1-a_{1}} \leq 2^{-1}$. This, with (2.15), shows that $s_{n} \leq(1 / 2)^{n}$ and so the lemma is proven.

A few remarks are as follows. First, it is clear that every irrational $x \in[0,1)$ has a unique expansion of the type of (2.1).

Second, we note that some particular cases of this type of continued fractions have been studied before. For example, by setting $q=1 / 2$ and $a_{k}=k$, the right-hand side of (1.8) gives the well-known continued fraction of Rogers and Ramanujan:

$$
\frac{q}{1+\frac{q^{2}}{1+\frac{q^{3}}{1+\cdots}}}
$$

Another example is the beautiful result due to Davison [7]. Let $a_{k}=F_{k}$, where $F_{k}$ is the $k$ th Fibonacci number. Davison showed that

$$
\frac{2^{-F_{1}}}{1+\frac{2^{-F_{2}}}{1+\frac{2^{-F_{3}}}{1+\cdots}}}=\frac{1}{2} \sum_{n \geq 1} 2^{-\lfloor n \phi\rfloor},
$$

where $\phi$ is the Golden Ratio and $\lfloor\cdot\rfloor$ denotes the floor function.

Third, we give an example. In terms of the continued fraction of the type of (1.8), we have

$$
\pi-3=\frac{2^{-2}}{1+\frac{2^{-0}}{1+\frac{2^{-1}}{1+\cdots}}}=[2,0,1,0,0,0,1,1,1,6, \ldots] .
$$

Here, in the first equality, we gave only the first three digits. In the second equality where we used the compact notation in (1.8), we gave the first ten digits.

Next, we prove the following lemma.

LEMMA 2.4. Let $c^{-1}=\log (4 / 3)$. The invariant probability density of the map $T$ is given by

$$
\rho(t)=\frac{c}{(1+t)(2+t)} .
$$

REMARK 2.5. As expected, the integral of $\rho$ is the first term of the right-hand side of (1.13), that is,

$$
\int_{0}^{t} \rho(s) d s=c \log \left(1+\frac{t}{2+t}\right) .
$$

Proof. To this end, we need to show that $\rho(t)$ defined in (2.20) is an eigenfunction of eigenvalue 1 of the Perron-Frobenius operator (see, e.g., [14, 15, 25])

$$
L_{T} \rho(t)=\sum_{s \in T^{-1}(t)} \frac{\rho(s)}{\left|T^{\prime}(s)\right|} .
$$


First, we note that

$$
T^{-1}(t)=\left\{\frac{2^{-k}}{1+t} ; k=0,1,2, \ldots\right\} .
$$

With this understood, we have, with $\gamma=1 / 2$,

$$
\begin{aligned}
L_{T} \rho(t) & =c \sum_{k=0}^{\infty} \frac{\gamma^{k}}{(1+t)^{2}} \rho\left(\frac{\gamma^{k}}{1+t}\right) \\
& =c \sum_{k=0}^{\infty} \frac{\gamma^{k+1}}{\left(t+1+\gamma^{k}\right)\left(t+1+\gamma^{k+1}\right)} \\
& =c \sum_{k=0}^{\infty} \frac{1}{t+1+\gamma^{k+1}}-\frac{1}{t+1+\gamma^{k}} \\
& =c\left(\frac{1}{t+1}-\frac{1}{t+2}\right) \\
& =\rho(t) .
\end{aligned}
$$

This proves the lemma.

3. Proof of Theorem 1.1. Here, we follow [23, pages 152-155]; see also [10, 17, 25]. First, by following the same trick that is used in the original Gauss-Kuzmin-Lévy problem, one can show that $\left\{G_{k}(t)\right\}$, defined in (1.12), satisfy a Kuzmin-type equation

$$
G_{k+1}(t)=\sum_{m=0}^{\infty} G_{k}\left(\gamma^{m}\right)-G_{k}\left(\frac{\gamma^{m}}{1+t}\right) .
$$

Just as in the original Gauss-Kuzmin-Lévy problem, it is easier to work with the derivative of $G_{k}(t)$. To this end, we observe that since the derivative of $G_{0}(t)=t$ is bounded in the unit interval, we can show by induction that the derivative of $G_{k}(t)$ is also bounded in the unit interval.

This allows us to differentiate (3.1) term-by-term, obtaining

$$
G_{k+1}^{\prime}(t)=\sum_{m \geq 0} \frac{\gamma^{m}}{(1+t)^{2}} G_{k}^{\prime}\left(\frac{\gamma^{m}}{1+t}\right) \text {. }
$$

Here, the prime denotes the derivative with respect to $t$. Next, we introduce $f_{k}(t)$ in such a way that

$$
G_{k}^{\prime}(t)=\frac{f_{k}(t)}{(1+t)(2+t)}
$$

In terms of $f_{k}(t),(3.2)$ can be written as

$$
f_{k+1}(t)=\sum_{m \geq 0} p_{m}(t) f_{k}\left(\frac{\gamma^{m}}{1+t}\right)
$$

where

$$
p_{m}(t)=\frac{\gamma^{m+1}(1+t)(2+t)}{\left(1+t+\gamma^{m}\right)\left(1+t+\gamma^{m+1}\right)}=\gamma^{m+1}+\frac{\Delta_{m}}{1+t+\gamma^{m}}-\frac{\Delta_{m+1}}{1+t+\gamma^{m+1}} .
$$


Here, $\Delta_{m}:=\gamma^{m}-\gamma^{2 m}$. Note that it follows from the definition of $p_{m}(t)$ that it is manifestly nonnegative for all $t \in[0,1)$ and for all natural numbers $m$. Also, we have used partial fraction decomposition in obtaining the second equality.

These formulae fit into the overall strategy as follows. Introduce a function $R_{k}(t)$ such that

$$
G_{k}(t)=c \log \left(1+\frac{t}{2+t}\right)+R_{k}\left(c \log \left(1+\frac{t}{2+t}\right)\right) .
$$

Here, $c$ is the constant in Theorem 1.1. Because $G_{k}(0)=0$ and $G_{k}(1)=1$, we have $R_{k}(0)=R_{k}(1)=0$. To prove the theorem, we have to show that

$$
R_{k}=O\left(q^{k}\right),
$$

where $0<q<1$. To achieve this goal, we proceed as follows. First, by comparing the derivatives of (3.3) and of (3.6), we obtain

$$
R_{k}^{\prime \prime}\left(c \log \left(1+\frac{t}{2+t}\right)\right)=\frac{(1+t)(2+t)}{c^{2}} f_{k^{\prime}}(t) .
$$

Next, by using (3.4), we can show that (the details will be given below)

$$
f_{k}^{\prime}(t)=O\left(q^{k}\right)
$$

for $0<q<1$. With (3.8), this implies $R_{k}^{\prime \prime}=O\left(q^{k}\right)$. Finally, by an interpolation formula

$$
R_{k}(t)=-\frac{t(1-t)}{2} R_{k}^{\prime \prime}(\xi),
$$

where $0<\xi<1$, we arrive at (3.7), and Theorem 1.1 is proven. All we need to do is to prove (3.9), as promised.

First, we note that from (3.4), we have

$$
f_{k+1}^{\prime}(t)=\sum_{m \geq 0} p_{m}^{\prime}(t) f_{k}\left(\frac{\gamma^{m}}{1+t}\right)-\sum_{m \geq 0} p_{m}(t) \frac{\gamma^{m}}{(1+t)^{2}} f_{k}^{\prime}\left(\frac{\gamma^{m}}{1+t}\right) .
$$

Our immediate goal is to express, by using (3.11), $f_{k+1}^{\prime}(t)$ in terms of $f_{k}^{\prime}(t)$. To this end, we need to rewrite the first sum in (3.11) as follows. By the second equality of (3.5) and partial summation, we have

$$
\begin{aligned}
\sum_{m \geq 0} p_{m}^{\prime}(t) f_{k}\left(\frac{\gamma^{m}}{1+t}\right) & =\sum_{m \geq 0}\left(\frac{\Delta_{m+1}}{\left(1+t+\gamma^{m+1}\right)^{2}}-\frac{\Delta_{m}}{\left(1+t+\gamma^{m}\right)^{2}}\right) f_{k}\left(\frac{\gamma^{m}}{1+t}\right) \\
& =\sum_{m \geq 0} \frac{\Delta_{m+1}}{\left(1+t+\gamma^{m+1}\right)^{2}}\left(f_{k}\left(\frac{\gamma^{m}}{1+t}\right)-f_{k}\left(\frac{\gamma^{m+1}}{1+t}\right)\right) .
\end{aligned}
$$

This, with the application of the mean value theorem to the difference

$$
f_{k}\left(\frac{\gamma^{m}}{1+t}\right)-f_{k}\left(\frac{\gamma^{m+1}}{1+t}\right)
$$


enables us to rewrite (3.11) as

$$
f_{k+1}^{\prime}(t)=\sum_{m \geq 0} \frac{\gamma^{m+1} \Delta_{m+1}}{(1+t)\left(1+t+\gamma^{m+1}\right)^{2}} f_{k}^{\prime}\left(\theta_{m}\right)-\sum_{m \geq 0} p_{m}(t) \frac{\gamma^{m}}{(1+t)^{2}} f_{k}^{\prime}\left(\frac{\gamma^{m}}{1+t}\right),
$$

with

$$
\frac{\gamma^{m+1}}{1+t}<\theta_{m}<\frac{\gamma^{m}}{1+t}
$$

Note that the right-hand side of (3.14) is written in terms of the derivative of $f_{k}(t)$ alone.

With the above understood, we proceed to compare the maximum of $f_{k+1}^{\prime}$ and that of $f_{k}^{\prime}$. Let $M_{j}$ be the maximum of $\left|f_{j}^{\prime}(t)\right|$ on $t \in[0,1]$. Then (3.14) implies

$$
M_{k+1} \leq M_{k} \cdot \max _{t \in[0,1]}\left|\sum_{m \geq 0} \frac{\gamma^{m+1} \Delta_{m+1}}{(1+t)\left(1+t+\gamma^{m+1}\right)^{2}}+\sum_{m \geq 0} p_{m}(t) \frac{\gamma^{m}}{(1+t)^{2}}\right| .
$$

We estimate the sums on the right-hand side of (3.16). First, we note that each term in the first sum in (3.16) is bounded; precisely, we have

$$
\frac{\gamma^{m+1} \Delta_{m+1}}{(1+t)\left(1+t+\gamma^{m+1}\right)^{2}} \leq \frac{\gamma^{2 m+2}}{\left(1+\gamma^{m+1}\right)^{2}}
$$

Next, observe that the function (cf. the second sum in (3.16))

$$
p_{m}(t) \frac{\gamma^{m}}{(1+t)^{2}}
$$

is decreasing for $t \geq 0$. Therefore, it attains its maximum at $t=0$. This leads to

$$
p_{m}(t) \frac{\gamma^{m}}{(1+t)^{2}} \leq \frac{\gamma^{2 m}}{\left(1+\gamma^{m}\right)\left(1+\gamma^{m+1}\right)} \leq \frac{\gamma^{2 m}}{\left(1+\gamma^{m+1}\right)^{2}} .
$$

These two observations allow us to rewrite inequality (3.16) as

$$
M_{k+1} \leq q M_{k}
$$

where

$$
q:=\left(1+\gamma^{2}\right) \sum_{m \geq 0} \frac{\gamma^{2 m}}{\left(1+\gamma^{m+1}\right)^{2}}=5 \sum_{m \geq 0} \frac{1}{\left(1+2^{m+1}\right)^{2}} .
$$

Since we have, for $m \geq 2$,

$$
\frac{1}{\left(1+2^{m+1}\right)^{2}} \leq \frac{1}{20}\left(\frac{1}{2}\right)^{m}
$$

therefore,

$$
q \leq 5\left(\frac{1}{9}+\frac{1}{25}+\frac{1}{20} \sum_{m \geq 2} \frac{1}{2^{m}}\right)=0.880555 \cdots<1 .
$$


This, with (3.20), implies $f_{k}^{\prime}(t)=O\left(q^{k}\right)$, that is, (3.9). This completes the proof of Theorem 1.1.

ACKNOWLEDGMENTS. I would like to thank my colleagues Chung-Hsien Sung and Douglas Woken for their support and encouragement. Also, I would like to thank the referees, whose comments were extremely valuable and helpful.

\section{REFERENCES}

[1] K. I. Babenko, On a problem of Gauss, Soviet Math. Dokl. 19 (1978), 136-140.

[2] R. M. Corless, Continued fractions and chaos, Amer. Math. Monthly 99 (1992), no. 3, 203215.

[3] P. Cvitanović, Circle maps: irrationally winding, From Number Theory to Physics (Les Houches, 1989) (M. Waldschmidt, P. Moussa, J. M. Luck, and C. Itzykson, eds.), Springer-Verlag, Berlin, 1992, pp. 631-658.

[4] K. Dajani and C. Kraaikamp, Generalization of a theorem of Kusmin, Monatsh. Math. 118 (1994), no. 1-2, 55-73.

[5] _ A Gauss-Kusmin theorem for optimal continued fractions, Trans. Amer. Math. Soc. 351 (1999), no. 5, 2055-2079.

[6] _ Ergodic Theory of Numbers, Carus Mathematical Monographs, vol. 29, Mathematical Association of America, Washington, DC, 2002.

[7] J. L. Davison, A series and its associated continued fraction, Proc. Amer. Math. Soc. 63 (1977), no. 1, 29-32.

[8] P. Flajolet, B. Vallée, and I. Vardi, Continued fractions from Euclid to the present day, preprint, 2000, http://www.lix.polytechnique.fr/Labo/Ilan.Vardi/publications.html.

[9] C. Ganatsiou, On a Gauss-Kuzmin type problem for piecewise fractional linear maps with explicit invariant measure, Int. J. Math. Math. Sci. 24 (2000), no. 11, 753-763.

[10] M. Iosifescu, A very simple proof of a generalization of the Gauss-Kuzmin-Lévy theorem on continued fractions, and related questions, Rev. Roumaine Math. Pures Appl. 37 (1992), no. 10, 901-914.

[11]__ On the Gauss-Kuzmin-Lévy theorem. I, Rev. Roumaine Math. Pures Appl. 39 (1994), no. 2, 97-117.

[12] __ On the Gauss-Kuzmin-Lévy theorem. II, Rev. Roumaine Math. Pures Appl. 40 (1995), no. 2, 91-105.

[13] _ On the Gauss-Kuzmin-Lévy theorem. III, Rev. Roumaine Math. Pures Appl. 42 (1997), no. 1-2, 71-88.

[14] M. Iosifescu and S. Grigorescu, Dependence with Complete Connections and Its Applications, Cambridge Tracts in Mathematics, vol. 96, Cambridge University Press, Cambridge, 1990.

[15] M. Iosifescu and C. Kraaikamp, Metrical Theory of Continued Fractions, Mathematics and Its Applications, vol. 547, Kluwer Academic Publishers, Dordrecht, 2002.

[16] A. Y. Khintchin, Continued Fractions, Dover, New York, 1997.

[17] D. E. Knuth, The Art of Computer Programming. Vol. 2, 2nd ed., Addison-Wesley Series in Computer Science and Information Processing, Addison-Wesley Publishing, Massachusetts, 1981.

[18] R. Kuzmin, Sur un problème de Gauss, Atti Congresso Bologna 6 (1932), 83-89 (French).

[19] P. Lévy, Sur les lois de probabilité dont dépendent les quotients complets et incomplets d'une fraction continue, Bull. Soc. Math. France 57 (1929), 178-194 (French).

[20] H. Nakada, On the invariant measures and the entropies for continued fraction transformations, Keio Math. Sem. Rep. (1980), no. 5, 37-44.

[21] H. Nakada, Sh. Ito, and S. Tanaka, On the invariant measure for the transformations associated with some real continued-fractions, Keio Engrg. Rep. 30 (1977), no. 13, 159-175. 
[22] G. J. Rieger, Über die mittlere schrittanzahl bei divisionsalgorithmen, Math. Nachr. 82 (1978), 157-180 (German).

[23] A. M. Rockett and P. Szüsz, Continued Fractions, World Scientific Publishing, New Jersey, 1992.

[24] T. A. Schmidt, Remarks on the Rosen $\lambda$-continued fractions, Number Theory with an Emphasis on the Markoff Spectrum (Provo, Utah, 1991) (A. D. Pollington and W. Moran, eds.), Lecture Notes in Pure and Appl. Math., vol. 147, Marcel Dekker, New York, 1993, pp. 227-238.

[25] F. Schweiger, Ergodic Theory of Fibred Systems and Metric Number Theory, Oxford Science Publications, Clarendon Press, Oxford University Press, New York, 1995.

[26] F. Schweiger and M. Waterman, Some remarks on Kuzmin's theorem for F-expansions, J. Number Theory 5 (1973), 123-131.

[27] P. Szüsz, Über einen kusminschen satz, Acta Math. Acad. Sci. Hungar. 12 (1961), 447-453 (German).

[28] S. Tanaka and S. Ito, On a family of continued-fraction transformations and their ergodic properties, Tokyo J. Math. 4 (1981), no. 1, 153-175.

[29] M. S. Waterman, A Kuzmin theorem for a class of number theoretic endomorphisms, Acta Arith. 19 (1971), 31-41.

[30] E. Wirsing, On the theorem of Gauss-Kusmin-Lévy and a Frobenius-type theorem for function spaces, Acta Arith. 24 (1974), 507-528.

Hei-Chi Chan: Mathematical Sciences Program, University of Illinois, Springfield, IL 62794-9243, USA

E-mail address: chan.hei-chi@uis.edu 


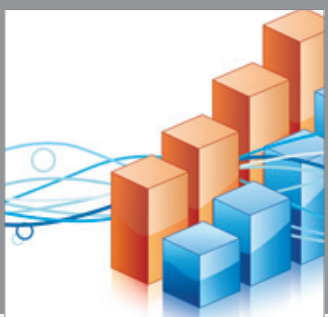

Advances in

Operations Research

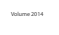

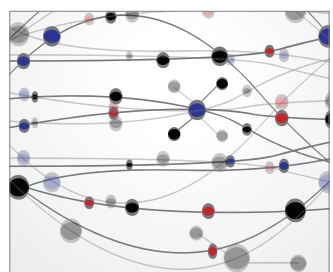

\section{The Scientific} World Journal
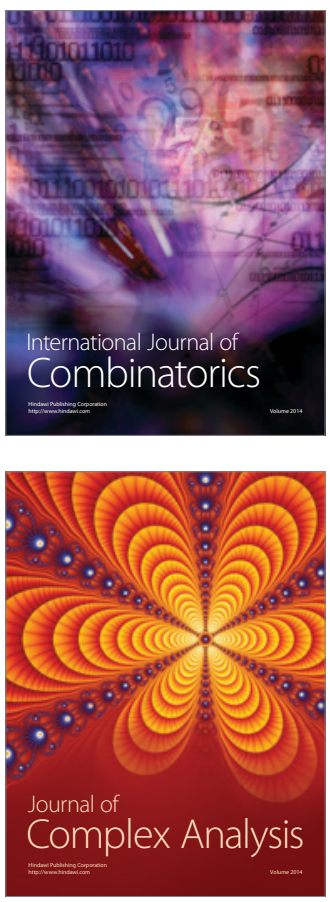

International Journal of

Mathematics and

Mathematical

Sciences
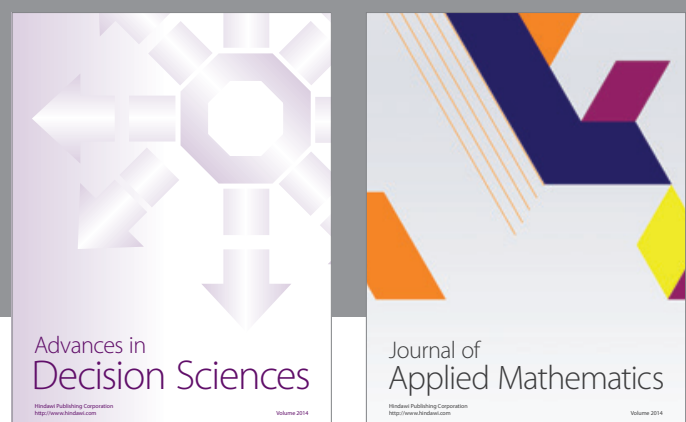

Journal of

Applied Mathematics
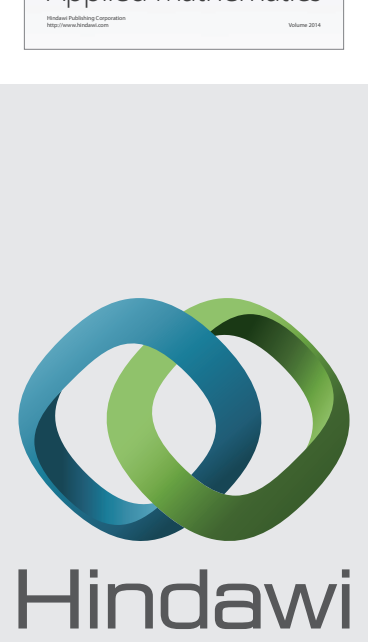

Submit your manuscripts at http://www.hindawi.com
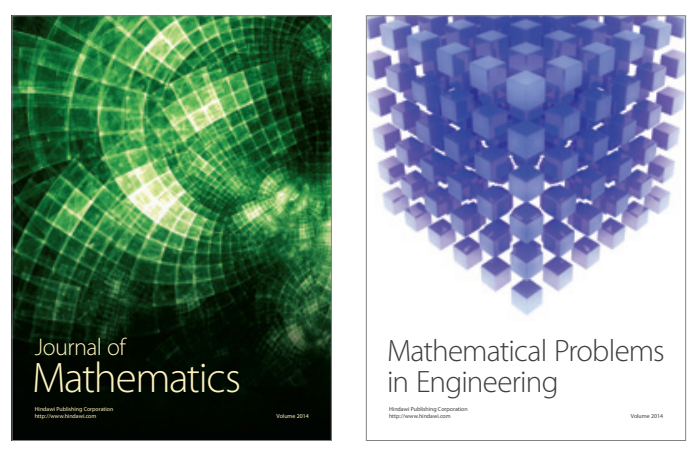

Mathematical Problems in Engineering
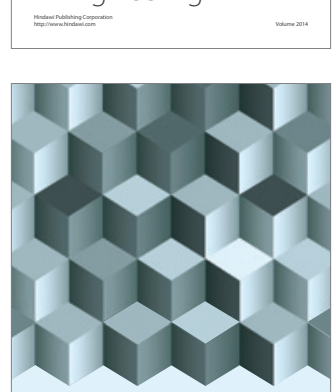

Journal of

Function Spaces
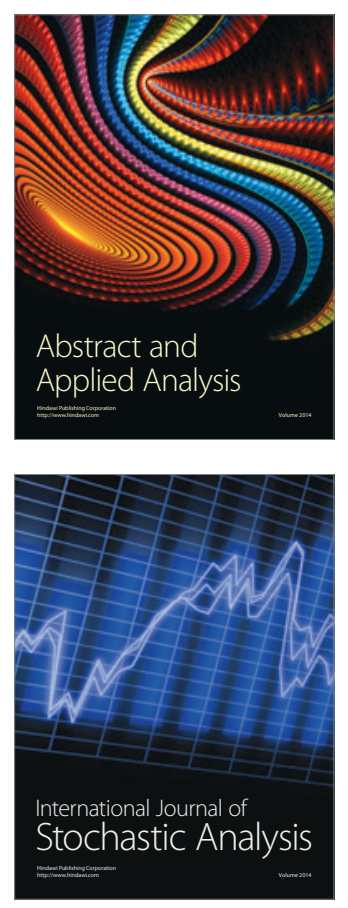

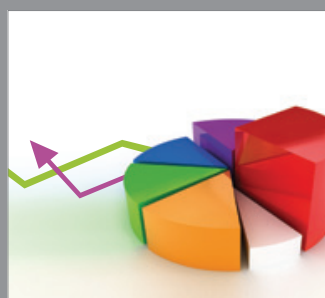

ournal of

Probability and Statistics

Promensencen
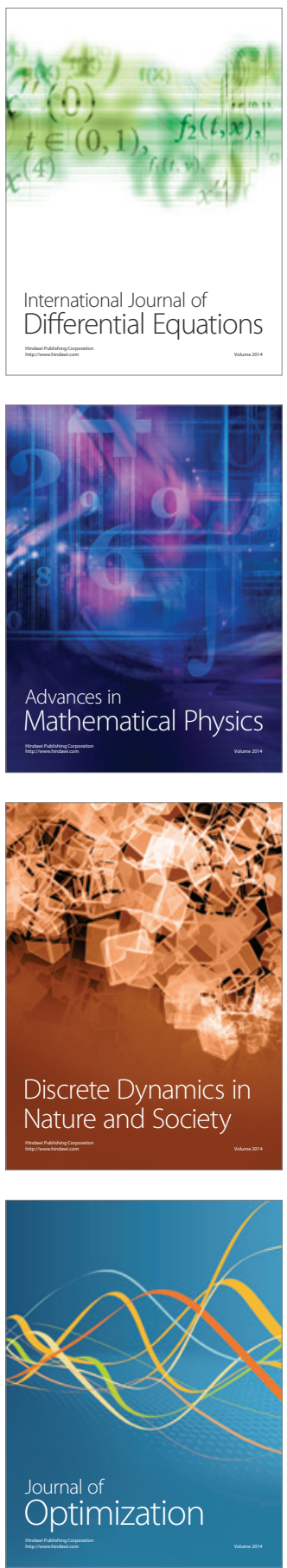